\title{
Bacterial Profiles of Subgingival Plaques in Periodontitis*
}

\author{
W. J. Loesche,† S. A. Syed, $\ddagger$ E. Schmidt§ and E. C. Morrison॥
}

Accepted for publication 22 January 1985

\begin{abstract}
IN THIS REPORT OVER 400 subgingival plaque samples taken from over 110 patients were examined microscopically and culturally for 30 bacterial parameters. The patients could be placed into six disease categories based upon clinical criteria. The bacterial profile of each clinical category was generally distinctive of that category. Periodontal patients who had been successfully treated and maintained had plaques that were populated by significantly higher proportions of Streptococcus sanguis, Actinomyces viscosus, A. odontolyticus and $S$. mutans and significantly lower proportions of $B$. gingivalis and spirochetes compared to the five untreated disease categories. The spirochetes were the overwhelming microbial type in the plaques of adult periodontitis (AP) patients, averaging about $45 \%$ of the microscopic count. The bacteriological results could not distinguish between ADA Type III and IV periodontitis, suggesting that the same type of infection was occurring in an active site in any AP patient. The patients designated as early onset periodontitis (EOP) differed from the other patients by their relative youth and by their significantly higher proportions of Bacteroides gingivalis and/or B. intermedius. Two types of EOP were recognized in which the most diseased variant was characterized by having an average of $49 \%$ spirochetes in the plaque. Four localized juvenile periodontitis (LJP) patients were notable in not having detectable $A$. actinomycetemcomitans. The data indicate that the various types of periodontitis, with the possible exception of LJP are specific anaerobic infections involving spirochetes and to a lesser extent $B$. gingivalis and $B$. intermedius.
\end{abstract}

Periodontal disease is the foremost cause of tooth loss in adult populations. ${ }^{1}$ In recent years considerable, but not unequivocal, data have appeared which suggest that some, if not all, forms of periodontal disease may be related either to the overgrowth or to the presence of one or more bacterial types in subgingival plaques removed from discrete tooth sites. ${ }^{2-5}$ The majority of this evidence is based upon cross-sectional association studies in which either the bacterial flora on diseased and nondiseased tooth surfaces are compared ${ }^{6-10}$ or the serum antibody titers to selected organisms in periodontally healthy or diseased individuals ${ }^{12-14}$ are compared.

Two general bacteriological approaches have been used to define the periodontopathic plaque flora. One is to subculture all or representative isolates from a given plaque sample and to perform as many taxo-

* This research was supported by Public Health service grants DE 06030 and DE 02731 from the National Institute of Dental Research.

† Professor of Dentistry. University of Michigan School of Dentistry, Ann Arbor, MI 48109.

$\ddagger$ Associate Professor of Dentistry, University of Michigan School of Dentistry.

$\S$ Visiting Assistant Professor, University of Michigan School of Dentistry.

|| Associate Research Scientist. University of Michigan School of Dentistry. nomic tests as are necessary in order to give the isolate a genus or species designation. This approach is necessary to define the plaque flora and has demonstrated an unusual amount of bacterial diversity within the plaque ecosystem ${ }^{8.10}$ with the concomitant recognition of many new species. ${ }^{15-17}$ However, only a few plaque samples can be so thoroughly characterized. Due to this, general statements concerning the role of any particular species in periodontal disease may be premature.

The other approach is to survey a large number of plaques for the presence of certain easily identifiable organisms, such as the black-pigmented Bacteroides (BPB), spirochetes, Capnocytophaga species, Fusobacterium nucleatum, Actinomyces viscosus and Actinobacillus actinomycetemcomitans, among others. This approach is informative if the identified organisms are either prominent members of the plaque flora, and/or if they contribute meaningfully to the health or disease potential of the plaque, as it permits microbial patterns to be recognized. If the sample size is large enough, statistical tests can be performed relative to both the tooth site and to the patient, thereby overcoming the objections raised when only sites nestled in the same patient are used in the analysis. ${ }^{18}$ Conversely, this approach would not identify a heretofore unknown periodontopathic organism unless by chance it was a con- 
spicuous organism on the various isolation media and was also enumerated.

The purpose of the present study was to examine a large number of subgingival plaques removed from untreated and successfully treated periodontal patients, in order to determine whether a characteristic bacterial profile could be identified that could be correlated with the clinical appearance of the periodontium in these patients. The patients were placed into six disease categories based upon clinical criteria and the bacterial profile of each clinical category was generally distinctive of that category.

\section{MATERIALS AND METHODS}

Classification of Periodontal Categories. Clinical and bacteriological data were collected from over 110 individuals who showed clinical and/or radiographic evidence of periodontal disease, as well as from 12 treated and maintained patients whose periodontal health had been stabilized for at least 1 year or more. All teeth, including molars with furcation involvement, were scored for pocket depth (probing depth) and loss of clinical attachment by one individual using procedures previously described. ${ }^{19}$ All information was used to assign the individuals to one of six clinical categories, using as a reference the American Dental Association (ADA) periodontal classification scheme. ${ }^{20}$ These clinical categories in turn served as the entities among which the bacteriological variables were compared.

Bacteriological Procedures. Subgingival plaque was usually removed from one pocket per quadrant in each individual. The site chosen appeared, from a clinical and radiographic examination, to be the most severely involved in each quadrant and was usually about molar teeth, i.e., 259 of 423 sampled sites were molars. The supragingival plaque about the sampled site was removed and discarded. The root surface was then scaled with a curette and the adherent plaque on the scaler tip was transferred to a vial containing $0.5 \mathrm{ml}$ of reduced transport fluid (RTF) without EDTA. ${ }^{21}$ The plaque samples were placed immediately into an anaerobic chamber and after dispersing for 20 seconds with a Vortex mixer, a $50-\mu$ l portion of each sample was removed for microscopic examination. This degree of dispersal separated most of the plaque aggregates without lysing the spirochetes. The remaining sample was diluted to $4 \mathrm{ml}$, sonically dispersed for 20 seconds with a Kontes sonifier, ${ }^{*}$ serially diluted in RTF and plated automatically with a spiral plater on a variety of media. In this manner microscopic and cultural counts were obtained on the same plaque sample.

The total anaerobic count, the count of BPB species, Capnocytophaga species, $F$. nucleatum and $A$ odontolyticus were obtained by identification of their distinctive colonies on enriched trypticase soy agar (ETSA). ${ }^{22}$

* Kontes Glass Co., Vineland, NJ.
Either all or representative colonies of BPB were identified as Bacteroides gingivalis, $B$. intermedius and $B$. melaninogenicus initially by their ability to use glucose, to hydrolyze esculin and to produce indole, and subsequently by rapid tests using chromogenic enzyme substrates. ${ }^{23.24} A$. viscosus and $A$. naeslundii were differentiated by catalase activity of colonies growing on a selective GMC $^{25}$ or CFAT agar. ${ }^{26}$ The total counts of facultative organisms, Streptococcus sanguis and S. mutans were obtained from growth on ETSA agar containing $2 \%$ sucrose and $20 \mu \mathrm{g} / \mathrm{ml}$ of metronidazole, which was incubated anaerobically. Veillonella colonies were enumerated on a medium containing MM10 base, minus blood but supplemented with lactate, vancomycin and a $0.004 \%$ bromocresol purple indicator. ${ }^{27}$

Ten $\mu \mathrm{l}$ of the aliquot removed for microscopic examination was placed on a glass slide, covered with a $22 \times 30 \mathrm{~mm}$ cover slip, sealed and viewed by dark-field microscopy. Either 200 organisms or the number of organisms in 20 high-power fields (hpf) were enumerated, depending on which event occurred first. The single cells were identified as spirochetes, selenomonads, vibrio-like motile rods, fusiforms, nonmotile rods or cocci. The spirochetes were further subdivided into large, intermediate and small-size. ${ }^{4}$

Statistical Analysis. The counts for each organism enumerated culturally and microscopically were recorded on forms suitable for computer processing. The proportions of these organisms were calculated by dividing the viable count of the specific organism by the total viable count on the ETSA agar plate in the case of the cultural data, and by dividing the microscopic count of the specific organism by the total microscopic count in the case of microscopic data. If there were less than 20 total colony-forming units (CFU) on the ETSA plate or in the microscopic count, no proportions were calculated. This was to eliminate distortions of the data which can occur when the denominator is very low. This was the case for microscopic counts in the treated and maintained patients.

The total counts and proportions of organisms in each clinical entity were statistically analyzed by an analysis of variance using the Scheffe test to compare between the clinical groups, and by the nonparametric Kruskal-Wallis test. The data were compared on a per patient basis by averaging the findings of 3 to 5 plaque samples removed from the patient so as to give an average value for each patient (total number $=112$ ) or by treating each plaque sample as a separate entity (total number $=423$ ). In the latter analysis it is assumed that the tooth site and not the patient is the meaningful biological point of reference.

\section{RESULTS}

The patients were placed into six distinct categories based upon clinical criteria (Table 1). The actual patient 
numbers vary somewhat in the tables as in a few patients either the microscopic or the cultural data were lost due to methodological problems. The 12 treated and maintained patients differed from the untreated patients in having fewer pockets greater than $3 \mathrm{~mm}$. The residual morbidity of their periodontal condition was evident in the high number of sites with attachment loss of 4 to $6 \mathrm{~mm}$ (Table 1). The treated and maintained patients included both ADA Type III and IV periodontal conditions. Four teenagers had the distinctive clinical appearance of localized juvenile periodontitis (LJP) with deep pockets and clinical attachment loss primarily about first molars.

A third group of patients was characterized by the clinical impression that an "active" process was ongoing. Similar patients have been reported by other investigators and given names such as "rapidly progressive," 28 "severe," 10 "advanced destructive" 14 or "generalized juvenile" 29 periodontitis. These patients will be referred to as early onset periodontitis (EOP) and could be subdivided into two groups called Types $\mathrm{A}$ and $\mathrm{B}$. The five Type A patients, despite being somewhat older, exhibited an earlier stage of periodontitis, as there were essentially no pockets or attachment loss greater than $6 \mathrm{~mm}$. They could be considered to be ADA Type II patients. In contrast, the Type B patients exhibited on the average 19 sites with pocket depths greater than 6 $\mathrm{mm}$ and 15 sites with attachment loss greater than 6 $\mathrm{mm}$ (Table 1). Bleeding upon probing was common in both types of patients.

A fourth group of patients was characterized as being "adult" periodontitis (AP). It was separated into two subgroups compatible with the ADA classification scheme for Type III and Type IV periodontitis. ${ }^{20}$ The Type IV patients were the oldest of the untreated patients and had significantly more sites with attachment loss greater than $6 \mathrm{~mm}$. They were the most common patient group encountered, comprising $64 \%$ of the patients in our study population. The EOP Type B patients could be considered, on the basis of probing characteristics, as being Type IV patients, but their youth, the clinical impression and the bacteriological profile (see below) indicated that they could be recognized as a separate entity.

The clinical characteristics of the sites sampled for the bacteriological analysis are given in Table 2. The depicted values are averages of 3 to 5 sites per patient and reflected the clinical distinctions between the six groups as described for the whole mouth (Table 1). Thus, the sampled pockets in the treated and maintained patients had probing depths that were significantly lower than the pocket depths in the five untreated categories (Table 2, Scheffe comparison $P=0.01$ ). The sampled pockets in the Type A EOP patients differed significantly from the sampled pockets in the Type B EOP patients in regard to pocket depth and attachment loss (Table 2). The deepest pockets were in the Type B EOP patients and in the LJP patients, whereas the sampled pockets with the greatest attachment loss were found in the Type IV patients. There were no significant differences in any of the measured clinical parameters between the Type B and Type IV pockets underlining the fact that these probing-derived parameters could not distinguish between the two types of patients.

Cultural studies revealed bacteriological differences between the EOP Types B and IV patients, in that the

Table 1

Clinical Characteristics of Patients Grouped According to Periodontal Morbidity and Age

\begin{tabular}{|c|c|c|c|c|c|c|}
\hline & \multicolumn{6}{|c|}{ Clinical Diagnosis of Periodontitis } \\
\hline & \multirow{2}{*}{$\begin{array}{l}\text { Treated and } \\
\text { maintained }\end{array}$} & \multicolumn{2}{|c|}{ Early onset } & \multicolumn{2}{|c|}{ Adult } & \multirow{2}{*}{ LJP } \\
\hline & & $\mathrm{A}$ & B & III & IV & \\
\hline No. of patients & 12 & 5 & 18 & 14 & 64 & 4 \\
\hline Age in years & $46 \pm 10^{*}$ & $30 \pm 12 \dagger$ & $22 \pm 5$ & $34 \pm 4 \dagger$ & $44 \pm 11 \dagger$ & $16 \pm 4 \dagger^{* * *}$ \\
\hline ADA class & III and IV & II & IV & III & IV & \\
\hline Total teeth & $26 \pm 2$ & $25 \pm 3$ & $26 \pm 4$ & $24 \pm 3$ & $24 \pm 4$ & $27 \pm 0.4$ \\
\hline \multicolumn{7}{|l|}{ Pocket depth† } \\
\hline$\leq 3 \mathrm{~mm}$ & $109 \pm 11 \dagger$ & $79 \pm 14$ & $63 \pm 18$ & $73 \pm 23$ & $54 \pm 22$ & $102 \pm 12$ \\
\hline $4-6 \mathrm{~mm}$ & $20 \pm 12$ & $45 \pm 24$ & $47 \pm 19$ & $41 \pm 13$ & $48 \pm 17$ & $24 \pm 13$ \\
\hline $7-9 \mathrm{~mm}$ & $0.3 \pm 0.5$ & $0.4 \pm 0.5$ & $19 \pm 10$ & $5 \pm 4$ & $16 \pm 14$ & $8 \pm 2$ \\
\hline$\geq 10 \mathrm{~mm}$ & 0 & 0 & $1 \pm 1$ & 0 & $1 \pm 2$ & 0 \\
\hline \multicolumn{7}{|c|}{ Attachment loss $\dagger$} \\
\hline$\leq 3 \mathrm{~mm}$ & $88 \pm 19 t$ & $107 \pm 8$ & $80 \pm 25$ & $68 \pm 37$ & $42 \pm 27$ & $129 \pm 20$ \\
\hline $4-6 \mathrm{~mm}$ & $38 \pm 19$ & $17 \pm 23$ & $35 \pm 24$ & $44 \pm 26$ & $48 \pm 17$ & $6 \pm 2$ \\
\hline $7-9 \mathrm{~mm}$ & $3 \pm 4$ & $0.2 \pm 0.4$ & $15 \pm 14$ & $7 \pm 4$ & $26 \pm 16$ & $0.3 \pm 0.6$ \\
\hline$\geq 10 \mathrm{~mm}$ & 0 & 0 & $1 \pm 2$ & 0 & $3 \pm 4$ & 0 \\
\hline
\end{tabular}

$\square$ Value in box is significantly different from all values in row.

+ Number of sites per patient. Value with ${ }^{* *}$ is significantly different from all other values in row with same superscript.

* Average \pm standard deviation. 
Table 2

Clinical Characteristics of Sampled Pocket Sites (Average Values) in Patients Grouped According to Whole-Mouth Clinical Criteria

\begin{tabular}{|c|c|c|c|c|c|c|}
\hline & \multicolumn{6}{|c|}{ Whole-mouth clinical diagnosis of periodontitis } \\
\hline & \multirow{2}{*}{$\begin{array}{l}\text { Treated and } \\
\text { maintained }\end{array}$} & \multicolumn{2}{|c|}{ Early onset } & \multicolumn{2}{|c|}{ Adult (ADA) } & \multirow{2}{*}{$\begin{array}{c}\text { Localized } \\
\text { juvenile }\end{array}$} \\
\hline & & A & B & III & IV & \\
\hline No. of patients & 12 & 5 & 18 & 14 & 64 & 4 \\
\hline $\begin{array}{l}\text { Pocket depth } \\
\quad(\mathrm{mm})\end{array}$ & $3.9 \pm 1^{*}$ & $4.9 \pm 1 \dagger$ & $7.0 \pm 1.6 \dagger$ & $5.4 \pm 1.2$ & $6.1 \pm 1.4$ & $6.8 \pm 0.8$ \\
\hline $\begin{array}{l}\text { Attachment loss } \\
(\mathrm{mm})\end{array}$ & $3.7 \pm 0.9$ & $3.0 \pm 0.8 \dagger$ & $6.1 \pm 2.1 \dagger$ & $5.6 \pm 0.8$ & $6.7 \pm 1.4$ & $4.5 \pm 1.6$ \\
\hline
\end{tabular}

$\square$ Value in row significantly different from all other values in row, Scheffe Test $P<0.05$.

* All values are an average of 3 to 5 pocket sites per patient.

$\uparrow$ Values in same row with same superscript are significantly different.

Table 3

Bacterial Characteristics of Plaque from Sampled Pocket Sites (Average Value per Patient)

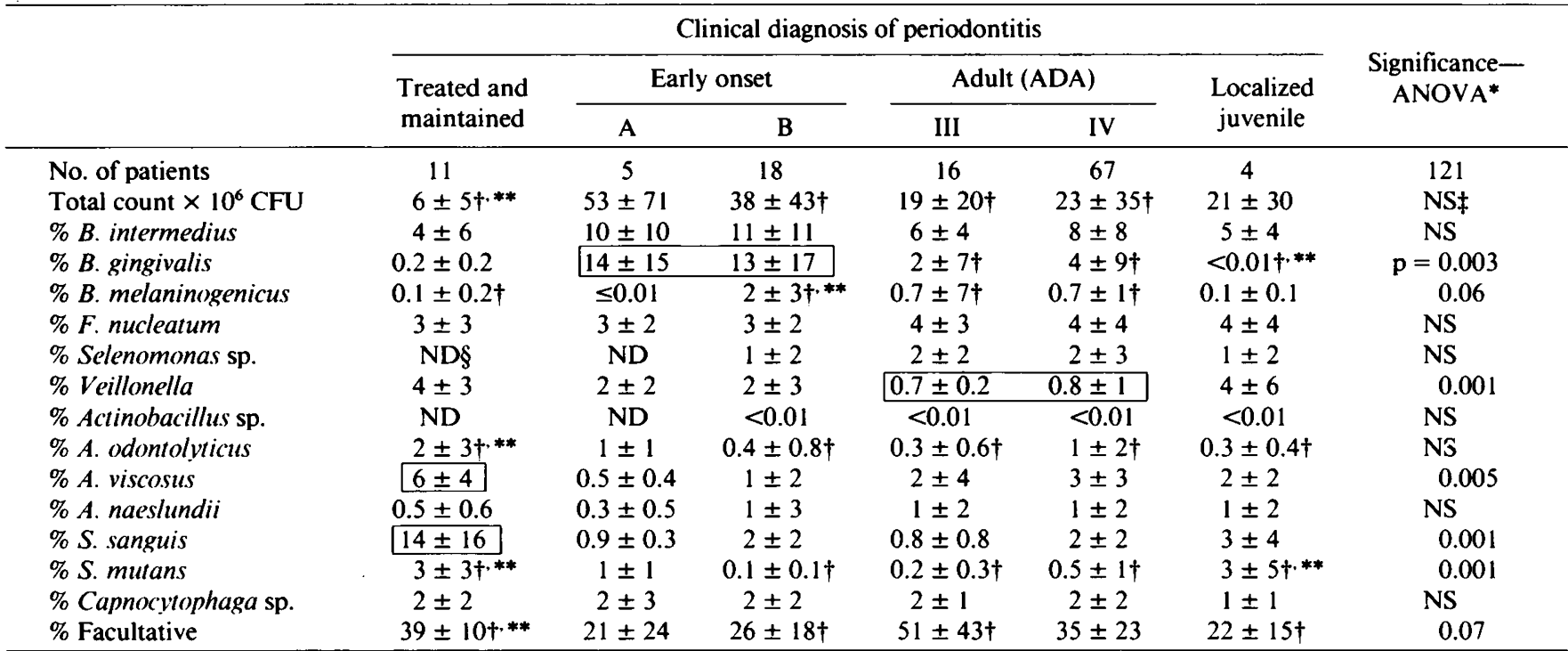

$\square$ Value is significantly different from all other values in row-Scheffe.

* Analysis of variance.

$\dagger$ Value with ${ }^{* *}$ is significantly different from other values in row with same superscript, i.e., $P<0.05$, Scheffe contrast.

$\ddagger$ NS, not significant.

$\S \mathrm{ND}$, not determined.

plaque removed from the Type B pockets had significantly higher proportions of $B$. gingivalis, $B$. melaninogenicus and Veillonella sp. relative to the pockets in the Type IV patients (Table 3 ). The high proportions of $B$. gingivalis appeared to be pathognomonic in the EOP patients, as both Types A and B plaques had significantly higher proportions of $B$. gingivalis relative to the four other clinical categories (Table 3). Types A and $B$ patients could be distinguished from each other microscopically in that the Type A plaque had significantly lower proportions of total spirochetes and small spirochetes, and significantly higher proportions of cocci (Table 4).

A unique bacterial profile was found in plaques removed from the treated and maintained patients, in that they differed in seven cultural parameters (Table 3 ) and in two microscopic parameters (Table 4) from the plaques removed from the other clinical categories.
In particular, the proportions of $A$. viscosus and $S$. sanguis were significantly higher than those found in any other clinical category. The proportions of $S . m u$ tans, A. odontolyticus and total facultative organisms were significantly higher than those values found in at least three of the other categories (Table 3 ). The proportions of spirochetes (Table 4 ) and $B$. gingivalis (Table 3 ) were significantly lower than those values found in the untreated categories. The spirochetes could only be detected in 12 of 28 plaques and $B$. gingivalis in 10 of 44 plaques examined. There was less plaque present in these pockets, as judged by the low total viable count (Table 3) and low numbers of bacteria per hpf (Table 4).

The plaque removed from the LJP patients was distinctive in not having any detectable $B$. gingivalis or $A$. actinomycetemcomitans isolates. This plaque tended to have higher proportions of $S$. mutans and lower pro- 
Table 4

Microscopic Characteristics of Plaque from Sampled Pocket Sites (Average Value per Patient)

\begin{tabular}{|c|c|c|c|c|c|c|c|}
\hline & \multicolumn{6}{|c|}{ Clinical diagnosis of periodontitis } & \multirow{3}{*}{$\begin{array}{c}\text { Significance- } \\
\text { ANOVA* }\end{array}$} \\
\hline & \multirow{2}{*}{$\begin{array}{l}\text { Treated and } \\
\text { maintained }\end{array}$} & \multicolumn{2}{|c|}{ Early onset } & \multicolumn{2}{|c|}{ Adult (ADA) } & \multirow{2}{*}{$\begin{array}{l}\text { Localized } \\
\text { juvenile }\end{array}$} & \\
\hline & & A & B & III & IV & & \\
\hline No. of patients & 7 & 4 & 14 & 16 & 65 & 4 & 110 \\
\hline Bacteria/hpft & $0.9 \pm 0.7$ & $36 \pm 29$ & $25 \pm 18$ & $16 \pm 10$ & $17 \pm 10$ & $27 \pm 31$ & $P=0.001$ \\
\hline \% Spirochetes & $12 \pm 9 \ddagger^{* * *}$ & $18 \pm 12$ & $49 \pm 18 \ddagger$ & $44 \pm 16 \pm$ & $49 \pm 20 \ddagger$ & $33 \pm 31 \ddagger$ & 0.001 \\
\hline$\%$ large & ND\$ & $2 \pm 3$ & $8 \pm 6$ & $4 \pm 5$ & $7 \pm 7$ & $6 \pm 7$ & $N S \|$ \\
\hline$\%$ intermediate & ND & $8 \pm 9$ & $13 \pm 6$ & $10 \pm 7$ & $12 \pm 8$ & $15 \pm 15$ & NS \\
\hline$\%$ small & ND & $8 \pm 3$ & $27 \pm 13$ & $30 \pm 15$ & $31 \pm 13$ & $12 \pm 15$ & 0.002 \\
\hline$\%$ Selenomonas & $4 \pm 5$ & $7 \pm 3$ & $7 \pm 5$ & $11 \pm 4$ & $10 \pm 10$ & $3 \pm 3$ & NS \\
\hline$\%$ Vibrio-like & $5 \pm 6$ & $4 \pm 1$ & $4 \pm 4$ & $3 \pm 3$ & $3 \pm 4$ & $3 \pm 3$ & NS \\
\hline$\%$ Motile & ND & ND & $1 \pm 2$ & $6 \pm 7$ & $3 \pm 3$ & ND & NS \\
\hline$\%$ Fusiforms & $9 \pm 5$ & $6 \pm 2$ & $5 \pm 4$ & $6 \pm 4$ & $5 \pm 5$ & $14 \pm 13$ & 0.01 \\
\hline$\%$ Rods & ND & $30 \pm 5$ & $19 \pm 13$ & $21 \pm 11$ & $20 \pm 10$ & $12 \pm 12$ & NS \\
\hline$\%$ Cocci & ND & $36 \pm 15$ & $14 \pm 15$ & $10 \pm 9$ & $11 \pm 10$ & $18 \pm 15$ & 0.002 \\
\hline
\end{tabular}

$\square$ Values in box significantly different from all other values in row.

* Analysis of variance.

$\dagger$ hpf, high power field.

$\ddagger$ Value with ${ }^{* *}$ is significantly different from other values in same row with superscript.

$\S N D$, not determined.

II NS, not significant.

portions of facultative organisms relative to the other disease categories.

The plaques removed from pockets in the AP patients (Types III and IV) were characterized by very high proportions of spirochetes (Table 4) and modest proportions of $B$. gingivalis (Table 3 ). The proportions of Veillonella species were low in the chronic periodontitis patients. None of the monitored bacteriological criteria could distinguish the Type III patient from the Type IV patient, suggesting that the bacterial infection in these pockets was comparable.

The statistical analyses were repeated using the plaque sample as the statistical entity, rather than the patient. Despite the fact that the number of observations increased about fourfold, there were few changes in the values of most parameters and in the number of differences between groups that were significant (data not shown). Among the latter, the cultural data revealed that the EOP Type B plaques had significantly higher proportions of $B$. intermedius than did the plaques found in the Type III, the LJP and the treated and maintained patients. Also, the proportions of $B$. intermedius in the plaques of the treated and maintained patients were significantly less than those found in the EOP and Type IV plaques.

These microbiological findings showed that among the monitored bacterial types or species, elevated proportions of spirochetes, $B$. gingivalis and to a lesser extent $B$. intermedius could be significantly associated with one or more types of periodontitis, excluding LJP. The data upon which this conclusion was based consisted of average values for each bacterial parameter compared across clinical categories with either the patient or the pocket site serving as the statistical entity. Not all patients were infected with these organisms, as can be observed by examining the frequency distribution of these bacterial types within each clinical category.

Spirochetes were the most common morphological type encountered in the study population, with the only exception being the treated and maintained patients. In these treated patients spirochetes were not detected in seven of 11 patients and when present, ranged from 11 to $27 \%$ of the microscopic count. Otherwise, the spirochetes were the dominant microscopic type found in 101 of 103 untreated patients averaging over $40 \%$ of the flora in $93 \%$ of the EOP Type B patients, in $75 \%$ of the Type III patients, and in $85 \%$ of the Type IV patients (Table 5).

B. gingivalis was not detected in about half the study population and not at all in the LJP patients (Table 6). None of the treated patients had $B$. gingivalis at levels above $1 \%$ of the viable count. Most of the EOP patients, however, had high proportions of $B$. gingivalis (Table 6 ). When $B$. gingivalis was not detected in these EOP patients, the plaques always had high proportions of $B$. intermedius. In fact, the pattern observed suggested that $B$. gingivalis and $B$. intermedius were mutually incompatible, a possibility that seemed to be verified from in vitro testing of these organisms, which showed that some $B$. gingivalis strains could inhibit the growth of $B$. intermedius and a few $B$. intermedius strains could inhibit certain strains of $B$. gingivalis (unpublished results).

$B$. intermedius was detected in 117 of 123 patients, and in this regard was about as frequently encountered as the spirochetes (Table 7). Unlike the spirochetes, $B$. intermedius was present in all the treated and maintained patients and in four of these patients was present in high proportions (Table 7). It also was a prominent 
Table 5

Frequency Distribution of Spirochetes in Patients of each Clinical Category

\begin{tabular}{|c|c|c|c|c|c|c|}
\hline \multirow[b]{2}{*}{ Disease category } & \multirow[b]{2}{*}{$\begin{array}{c}\text { No. of } \\
\text { patients }\end{array}$} & \multicolumn{5}{|c|}{$\%$ of Patients in whom spirochetes were } \\
\hline & & $\begin{array}{c}\text { Not } \\
\text { detected }\end{array}$ & $\begin{array}{c}<10 \% \text { of } \\
\text { micro- } \\
\text { scopic } \\
\text { count* }\end{array}$ & $\begin{array}{c}10-20 \% \\
\text { of } \\
\text { micro- } \\
\text { scopic } \\
\text { count }\end{array}$ & $\begin{array}{c}21-40 \% \\
\text { of } \\
\text { micro- } \\
\text { scopic } \\
\text { count }\end{array}$ & $\begin{array}{c}>40 \% \text { of } \\
\text { micro- } \\
\text { scopic } \\
\text { count* }\end{array}$ \\
\hline Treated and maintained & 11 & 64 & 0 & 9 & 27 & 0 \\
\hline EOP† Type A & 4 & 0 & 0 & 50 & 0 & 50 \\
\hline Type B & 14 & 0 & 0 & 0 & 7 & 93 \\
\hline APt III & 16 & 0 & 0 & 6 & 19 & 75 \\
\hline IV & 65 & 0 & 3 & 0 & 12 & 85 \\
\hline $\mathbf{L J P}+$ & 4 & 25 & 0 & 25 & 50 & 0 \\
\hline
\end{tabular}

* Patients were placed in the percentage range that corresponded with the highest percentage found in any single site in that mouth, i.e., if 4 sites in one patient were $5 \%, 15 \%, 22 \%$ and $42 \%$ spirochetes, the patient was placed in the $>40 \%$ column.

$\dagger$ EOP, early onset periodontitis; AP, adult periodontitis; LJP, localized juvenile periodontitis.

Table 6

Frequency Distribution of $B$. gingivalis in Patients for each Disease Category

\begin{tabular}{ccccc}
\hline \multirow{2}{*}{ Disease category } & No. of & \multicolumn{2}{c}{$\%$ of patients in whom $B$. gingivalis was } \\
\cline { 3 - 5 } & patients & $\begin{array}{c}\text { Not } \\
\text { detected }\end{array}$ & $\begin{array}{c}\leq 10 \% \text { of } \\
\text { viable count }\end{array}$ & $\begin{array}{c}>10 \% \text { of } \\
\text { viable count* }\end{array}$ \\
\hline Treated and maintained & 14 & 42 & 58 & 0 \\
EOP† Type A & 5 & 20 & .0 & 80 \\
Type B & 18 & 39 & 0 & 61 \\
APt III & 17 & 65 & 29 & 6 \\
IV & 67 & 51 & 28 & 21 \\
LJP† & 4 & 100 & 0 & 0
\end{tabular}

* If one or more sites in a patient had $>10 \% \mathrm{~B}$. gingivalis then the patient was counted as having $>10 \%$ B. gingivalis.

† EOP, early onset periodontitis; AP, adult periodontitis; LJP, localized juvenile periodontitis.

Table 7

Frequency Distribution of $B$. intermedius in Patients of each Clinical Category

\begin{tabular}{lcccc}
\hline \multirow{2}{*}{ Disease category } & No. of & \multicolumn{3}{c}{$\%$ of Patients in whom $B$. intermedius was } \\
\cline { 3 - 5 } & patients & $\begin{array}{c}\text { Not } \\
\text { detected }\end{array}$ & $\begin{array}{c}\leq 10 \% \text { of } \\
\text { viable count }\end{array}$ & $\begin{array}{c}>10 \% \text { of } \\
\text { viable count* }\end{array}$ \\
\hline Treated and maintained & 12 & 0 & 67 & 33 \\
EOPt Type A & 5 & 20 & 0 & 80 \\
Type B & 18 & 22 & 11 & 67 \\
APt III & 17 & 0 & 53 & 47 \\
IV & 67 & 3 & 42 & 55 \\
LJP† & 4 & 0 & 50 & 50 \\
\hline
\end{tabular}

* If one or more sites in a patient had $>10 \% \mathrm{~B}$. intermedius, then the patient was counted as having $>10 \%$ B. intermedius.

† EOP, early onset periodontitis; AP, adult periodontitis; LJP, localized juvenile periodontitis.

organism in two of the LJP patients. The EOP group was distinctive in having patients who were either highly infected with $B$. intermedius or were not detectably colonized by this organism (Table 7).

\section{DISCUSSION}

The present study of over 400 subgingival plaque samples removed from over 100 untreated periodontal patients and 12 treated and maintained patients indi- cate that sufficient microbial parameters exist which can supplement the clinical parameters to allow the delineation of at least 6 categories of periodontal health and disease.

The treated and maintained group of patients had the most distinctive bacteriological profile in that their plaques were populated by significantly higher proportions of $S$. sanguis, A. viscosus, $A$. odontolyticus and $S$. mutans and significantly lower proportions of $B$. gin- 
givalis and spirochetes compared to the five untreated disease categories (Tables 3-7). This combination of elevated proportions of facultative, plaque-forming bacteria and decreased proportions of anaerobic bacteria and spirochetes would seem to constitute the profile of a nondisease-associated plaque, the existence of which was postulated by the specific plaque hypothesis. ${ }^{2}$ The low proportions or absence of spirochetes and the high proportions of cocci and rods have been described in previous microscopic studies of plaque removed from relatively healthy sites ${ }^{4.30}$ and has been shown to be the flora which established following successful treatment. $^{31,32}$

The present study thus confirmed the previous findings and extended them by adding cultural studies, which identified some of the rods and cocci as $A$. viscosus, $A$. odontolyticus, $S$. sanguis and $S$. mutans. Species such as $S$. sanguis, $A$. viscosus and $S$. mutans are plaque formers in the supragingival domain, ${ }^{33}$ and the latter two species have been associated with root surface caries. ${ }^{34} \mathrm{~A}$. viscosus has been associated with gingivitis in the experimental gingivitis model ${ }^{35}$ and possesses in vitro both mitogenic and antigenic activity. ${ }^{36,37}$ Thus, the proportional ascendency of these species in nondisease-associated plaques would appear to contradict certain evidence implicating these organisms as odontopathogens.

Evidence of microbial behavior in vitro or in the supragingival domain, however, does not predict that microbe's behavior in another environment, such as the subgingival domain. It is possible that $S$. sanguis and $A$. viscosus, through the production of soluble factors such as hydrogen peroxide and bacteriocins, could reduce the plaque levels of presumably more virulent anaerobic organisms, such as the spirochetes. Also, the mitogenic and antigenic components of $S$. sanguis, A. viscosus and similar organisms could elicit the particular $\mathrm{T}$ lymphocyte response that is associated with a nonprogressive early lesion. ${ }^{38.39}$ In this sense, these nondisease-associated organisms may prime the host $\mathrm{T}$ cell response in such a way that a tissue-destroying $B$ cell infiltration ${ }^{39-41}$ does not occur.

The four LJP patients were notable in not having detectable $A$. actinomycetemcomitans. While $A$. actinomycetemcomitans can be significantly associated with $\mathrm{LJP},{ }^{42}$ cases have been described where it cannot be isolated. ${ }^{43}$ The LJP patients had no detectable $B$. gingivalis but were colonized by $B$. intermedius. Several investigators have reported spirochetes as undetectable, ${ }^{44}$ or low ${ }^{9}$ in LJP patients. This heterogeneity was observed in the present patients, as in two patients no spirochetes could be detected (Table 5).

The bacterial profile of the subgingival plaques in the AP individuals was rather uniform in that plaques removed from the Types III and IV patients were bacteriologically comparable in having high proportions of spirochetes, moderate proportions of $B$. inter- medius and low proportions of $B$. gingivalis and Veillonella (Tables 3 and 4). As the bacteriological sites were selected as the most diseased site per quadrant and had similar clinical morbidities (Table 2), the bacteriological findings probably reflect that the same type of infection was occurring in an active site in any AP patient. The quantification of the clinical morbidity, as is done by the ADA Types III and IV classification system did not seem to provide any diagnostic help in differentiating between types of infections within this large group of AP patients.

The spirochetes were the overwhelming microbial type in the plaques of our AP patients. This agrees with dark-field and electron-microscopic studies by Listgarten and his colleagues ${ }^{4.44}$ and phase-contrast microscopic observations by Keyes and Rams, ${ }^{45}$ which collectively make an impressive argument that spirochetes are pathognomonic in periodontal disease. ${ }^{46}$ One spirochete, Treponema denticola possesses a trypsin-like enzyme which could be a virulence mechanism in the periodontal pocket ${ }^{23}$ and produces a soluble factor which causes a dose-dependent inhibition of murine and human fibroblast proliferation. ${ }^{47}$

Against this background of a spirochetal infection, other plaque organisms, such as BPB, may occasionally overgrow, thereby causing perhaps a more "acute" infection. For example, in one investigation BPB were isolated from seven to eight plaques removed from eight patients aged 34 to 48 years at levels that averaged $32 \%$ of the cultivable flora. " Other investigators have associated $B$. gingivalis ( $B$. asaccharolyticus) with deep pockets, ${ }^{7}$ marked gingival inflammation ${ }^{7}$ and ADA Types III and IV. ${ }^{6}$ In these studies the age range of the patients was from 24 to 66 years, so that it is possible that some of these patients would have resembled our EOP patients. These studies reported that $B$. gingivalis was present in 40 to $55 \%$ of the examined pockets, a finding that was similar to that which was observed in the EOP patients (Table 6). B. intermedius was present in most individuals in both disease-associated and nondisease-associated plaques, ${ }^{6,7}$ but it can also be found in high proportions in the absence of $B$. gingivalis in adults with moderate to severe bone breakdown. ${ }^{48}$

The patients designated as early onset periodontitis (EOP) differed from the other patients by their relative youth and by their significantly higher proportions of $B$. gingivalis and/or $B$. intermedius. Young adult patients with a similar clinical appearance have been described in the recent literature and have been given descriptive terms, such as rapidly progressive periodontitis, ${ }^{28}$ severe periodontitis, ${ }^{10}$ advanced destructive periodontitis, ${ }^{14}$ postjuvenile periodontitis ${ }^{49}$ and generalized juvenile periodontitis. ${ }^{29}$ These epithets were deemed less descriptive than "early onset" for the following reasons:

The designation "rapidly progressive" requires that at least two clinical examinations be made over time in 
order to document the rapid progression of periodontal destruction. Most patients with this periodontal condition are not likely to have been observed thusly, so that it is doubtful that many clinicians or researchers could validly make this diagnosis. The terms "severe" and "advanced destructive" are appropriate, descriptive terms of the clinical morbidity observed, but do not convey a sense of the patient's youth. "Post juvenile" and "generalized juvenile" do convey the young age of the patient but suggest that the condition is or may be a sequela of LJP. for which currently no solid evidence exists.

Early onset periodontitis was recently used in referring to the periodontal findings in seven siblings who had either rapidly progressive periodontitis or juvenile periodontitis and in whom Bacteroides species were prominent members of the cultivable plaque flora. ${ }^{50}$ This descriptor seemed appropriate for our patients, as the designation "early onset" could represent both an early stage of periodontitis as seen in our Group A patients, as well as an early age of onset of periodontitis as seen in our Group B patients. EOP thus is a collective descriptor that can accommodate most, if not all, the periodontal conditions seen in young individuals that cannot be diagnosed as LJP.

That EOP represents more than one bacterial infection is amply demonstrated by the present findings and the reports of others. ${ }^{8.10}$ Our Type A patients had primarily a $B$. gingivalis infection with moderate proportions of $B$. intermedius and spirochetes, high proportions of cocci and no detectable $A$. actinomycetemcomitans. Our Type B patients had very high proportions of spirochetes and either high proportions of $B$. gingivalis or $B$. intermedius and no detectable $A$. actinomycetemcomitans. Tanner et al. ${ }^{8}$ isolated high proportions of $B$. gingivalis from four sites in two young individuals who exhibited "widespread destructive lesions involving most of the dentition." These sites also harbored high proportions of $A$. actinomycetemcomitans in their plaques. A third patient, who resembled the other two and our Type B patients, clinically had high proportions of $B$. gingivalis but no detectable $A$. actinomycetmcomitans. Spirochetes were not monitored. Moore et al. ${ }^{10}$ sampled 34 affected pocket sites in 21 patients whose clinical description resembled that of our Group B patients. They found a complex flora containing 146 bacterial taxa, but no $B$. gingivalis. Instead, $F$. nucleatum, Eubacterium timidum, E. nodatum, Lactobacillus minutus, B. intermedius and two treponemes were found in elevated proportions. $E \boldsymbol{U}$ bacterium and Lactobacillus species would not be identified by our protocol, but $F$. nucleatum was, and could not be associated with EOP (Tables 3 and 5).

Studies which monitor antibody titers in the peripheral blood against a panel of putative periodontal pathogens implicate $B$. gingivalis in EOP. Mouton et al. ${ }^{12}$ found that $50 \%$ of the individuals in a generalized juvenile periodontitis group had very high levels of IgG antibodies to $B$. gingivalis ( $B$. asaccharolyticus). Taubman et al. ${ }^{13}$ reported that the level of serum IgG antibody to $B$. gingivalis was higher in generalized juvenile periodontitis patients than in adult periodontitis patients. Ebersole et al. ${ }^{14}$ showed that $43 \%$ of the sera from a group of 62 patients categorized as advanced destructive periodontitis had high IgG antibodies to $B$. gingivalis and that a substantial proportion of these patients exhibited high antibody responses to both $B$. gingivalis and $B$. intermedius.

These bacteriological and immunological studies make a cogent argument for a role of $B$. gingivalis and possibly $B$. intermedius in some EOP patients. However, this role is clearly shared with, or may even be preempted in the Type B patient by the spirochetes. The spirochetes were present at levels greater than $40 \%$ of the flora in 13 of 14 Type B patients and averaged $30 \%$ in the other patient. The universality and magnitude of this spirochetal infection would make it the common bacterial denominator in Type B patients and would make these patients bacteriologically similar to the AP patients. Other investigators have reported that spirochetes averaged $56 \%$ of the flora in plaques removed from 8 post juvenile periodontitis patients, ${ }^{49}$ and $57 \%$ of the flora in plaques removed from $8 \mathrm{~mm}$ or deeper pockets in 22 patients between the ages of 21 and $28 .^{30}$ Thus, spirochetes, and not BPB or A. actinomycetemcomitans, are the organisms most typical of the Type B, EOP patient.

If this is so, then the recognition of the Type $A$ patients is of some interest, as in these patients the $B$. gingivalis- $B$. intermedius infection was comparable to the spirochetal infection in terms of microbial numbers and frequency of occurrence (Tables 4-7). The EOP Type A patients were initially classified as ADA Type II patients on the basis of probing depths and clinical attachment loss (Table 1), but because of the bacteriological findings were placed into the EOP category on the assumption that the Type A patient represented an early stage of a periodontal infection, involving primarily BPB, that occurred prior to a spirochetal overgrowth. It would appear from examining the other disease categories that as the spirochetes became the dominant entity in the plaque flora, the pockets were deeper (Table 2), and that the incidence of a detectable $B$. gingivalis colonization declined both in the EOP Type B and AP Types III and IV patients (Tables 5 and 6). This suggests that the ecological niche occupied by $B$. gingivalis is taken over by the spirochetes or some other unidentified species, either by an active process, i.e., bacterial antagonism, or by environmental conditions, i.e., low Eh, low $\mathrm{pO}_{2}{ }^{51}$ that favor the spirochetes or by host antibodies against $B$. gingivalis. ${ }^{12-14}$

Thus, a periodontopathic succession may exist in the subgingival plaques as $B$. gingivalis may be replaced by spirochetes as the periodontal condition progresses 
from a Type A-EOP to a Type B-EOP and later, on to AP. Diagnosis and treatment of the Type A-EOP patient might interrupt this sequence. The occasional occurrence of $B$. gingivalis in high proportions in plaques taken from AP patients (Table 6) could coincide with the asynchronous multiple bursts of periodontal destruction that have been observed in untreated periodontal patients. ${ }^{52.53}$ If this is so, then $B$. gingivalis could be the microbial indicator of acute infections whereas the spirochetes could be indicators of chronic infections.

The present study indicates that the various types of periodontitis, with the possible exception of LJP, are specific anaerobic infections involving spirochetes and to a lesser extent BPB. This could then explain the success of metronidazole, an antimicrobial that has a spectrum of activity limited to anaerobic bacteria, in the treatment of deep pockets highly infected with spirochetes. 5.32 .54

\section{ACKNOWLEDGMENTS}

Janice Stoll and Natalie Grossman assisted in the bacteriological aspects of this investigation. Neal Wright assisted in the computer data management.

\section{REFERENCES}

1. Page, R. C., and Schroeder, H. E.: Periodontitis in Man and other Animals, A Comparative Revien', 330 p. Basel, S. Karger, 1982.

2. Loesche, W. J.: Chemotherapy of dental plaque infections. Oral Sci Rev 9: 63, 1976.

3. Socransky, S. S., Tanner, A. C. R., Haffajee, A., et al.: Present status of studies on the microbial etiology of periodontal diseases. Host Parasite Interactions in Periodontal Disease. R. J. Genco and S. E. Mergenhagen (eds), pp 1-12. Washington, DC, American Society Microbiology, 1982.

4. Listgarten, M. A., and Hellden, L.: Relative distribution of bacteria at clinically healthy and periodontally diseased sites in humans. J Clin Periodontol 5: 115, 1978.

5. Loesche, W. J., Syed, S. A., Morrison. E. C., et al.: Metronidazole in periodontitis. I. Clinical and bacteriological results after 15 to 30 weeks. J Periodontol 55: 325, 1984.

6. Spiegel. C. A., Hayduk, S. E., Minah, G. E., and Krywolap, G. N.: Black-pigmented bacteroides from clinically characterized periodontal sites. $J$ Periodont Res 14: 376, 1979.

7. White, D., and Mayrand, D.: Association of oral bacteroides with gingivitis and adult periodontitis. J Periodont Res 16: 259, 1981.

8. Tanner, A. C., Haffer, C., Bratthall, G. T., et al.: A study of the bacteria associated with advancing periodontitis in man. J Clin Periodontol 6: 278, 1979.

9. Newman, M. G., and Socransky, S. S.: Predominant cultivable microbiota in periodontosis. $J$ Periodont Res 12: 120, 1977.

10. Moore, W. E. C., Holdeman, L. V., Smibert, R. M., et al.: Bacteriology of severe periodontitis in young adult humans. Infect Immun 38: $1137,1982$.

11. Slots, J.: The predominant cultivable microflora of advanced periodontitis. Scand J Dent Res 85: 114, 1977.

12. Mouton. C., Hammond, P. G., Slots, J., and Genco, R. J.: Serum antibodies to oral Bacteroides asaccharolyticus (Bacteroides gingivalis) relationship to age and periodontal disease. Infect Immun 31: 182,1981 .

13. Taubman, M. A., Ebersole, J. L. and Smith D. J.: Association between systemic and local antibody and periodontal diseases. HostParasite Interactions in Periodontal Disease. R. J Genco and S. E. Mergenhagen (eds). pp. 283-290. Washington, DC, American Society for Microbiology, 1982.

14. Ebersole, J. L., Taubman, M. A., Smith, D. J., and Frey, D. E.: Human immune response to oral microorganisms. III. Serological classification and systemic antibody response to Bacteroides species. Infect Immun, in press.

15. Holdeman, L. V., and Johnson, J. L.: Description of Bacteroides loescheii sp. nov. and emendation of the descriptions of Bacteroides melaninogenicus (Oliver and Wherry) Roy and Kelly 1939 and Bacteroides denticola Shah and Collins 1981. Int J Syst Bacteriol 32: $399,1982$.

16. Leadbetter, E. R., Holt, S. C., and Socransky, S. S.: Capnocytophaga: new genus of Gram-negative gliding bacteria. I. General characteristics, taxonomic considerations and significance. Arch $\mathrm{Mi}$ crobiol 122: 9, 1979.

17. Tanner, A. C. R., Badger, S., Lai, C.-H., et al.: Wolinella gen. nov., Wolinella succinogenes (Vibrio succinogenes Wolin et al.) comb. nov. and description of Bacteroides gracilis sp. nov., Wolinella recta sp. nov., Campylobacter concisus sp. nov. and Eikenella corrodens from humans with periodontal disease. Int J Syst Bacteriol 31: 432 , 1981.

18. Cohen, M. E., and Cecil, J. E.: Intraclass correlations and the application of analysis of variance to dental data. J Dent Res 62: 332, 1983.

19. Ramfjord, S. P., Knowles, J. W., Nissle, R. R., et al.: Longitudinal study of periodontal therapy. J Periodontol 44: 66, 1973.

20. Schluger, S., Yuodelis, R. A., and Page, R. C.: Periodontal Disease, 737 pp. Philadelphia, Lea and Febiger, 1977.

21. Loesche, W. J., and Syed, S. A.: The predominant cultivable flora of carious plaque and carious dentin. Caries Res 7: 201, 1973.'

22. Syed, S. A., Svanberg, M., and Svanberg, G.: The predominant cultivable flora of gingivitis associated plaque of beagle dogs. $J$ Periodont Res 15: 123, 1980.

23. Laughon, B. E., Syed, S. A., and Loesche, W. J.: API-ZYM system for identification of Bacteroides sp., Capnocytophaga sp. and spirochetes of oral origin. J Clin Microbiol 15: 97, 1982.

24. Laughon, B. E., Syed, S. A., and Loesche, W. J.: Rapid identification of Bacteroides gingivalis. J Clin Microbiol 15: 345, 1982.

25. Kornman, K. S., and Loesche, W. J.: New medium for isolation of Actinomyces viscosus and Actinomyces naeslundii from dental plaque. J Clin Microbiol 7: 514, 1978.

26. Zylber, L. J., and Jordan, H. V.: Development of a selective medium for detection and enumeration of Actinomyces viscosus and Actinomyces naes/undii in dental plaque. J Clin Microbiol 15: 253, 1982.

27. Loesche, W. J., Eklund, S., Earnest, R., and Burt, B.: Longitudinal investigation of bacteriology of human fissure decay: epidemiological studies in molars shortly after eruption. Infect Immun 46: $765,1984$.

28. Page, R. C., Altman, L. C., Ebersole, J. L., et al.: Rapidly progressive periodontitis. A distinct clinical condition. $J$ Periodontol 54: $197,1983$.

29. Genco, R. J., Slots, J., Mouton, C., and Murray, P.: Systemic immune responses to oral organisms. D. W. Lambe, Jr., R. J. Genco, and K. J. Mayberry-Carson (eds), Anaerobic Bacteria: Selected Topics, pp 277-293. New York, Plenum Publishing Corp., 1980.

30. Lindhe, J., Liljenberg, B., and Listgarten, M.: Some microbiological and histopathological features of periodontal disease in man J Periodontol 51: 264, 1980.

31. Listgarten, M. A., Lindhe, J., and Hellden, L.: Effect of tetracycline and/or scaling on human periodontal disease. J Clin Periodontol 5: 246, 1978.

32. Lindhe, J., Liljenberg, B., Adielson, B., and Borjesson, I.: Use of metronidazole as a probe in the study of human periodontal disease. J Clin Periodontol 10: 100, 1983.

33. Loesche, W. J.: Dental Caries: A Treatable Infection, 558 pp. Springfield, Charles C Thomas, 1982.

34. Syed, S. A., and Loesche, W. J.: Predominant cultivable flora 
isolated from human root surface caries plaque. Infect Immun 11: $727,1975$.

35. Loesche, W. J., and Syed, S. A.: Bacteriology of human experimental gingivitis. II. Effect of plaque and gingivitis scores. Infect Immun 21: 830, 1978.

36. Lehner, T.: Immunological responses to bacterial plaque in the mouth. CIBA Foundation Symposium, 26-28 April (46), pp 135154.

37. Burckhardt, J. J., Guggenheim, B., and Hefti, A.: Are $A c$ tinomyces viscosus antigens B cell mitogens? J Immunol 118: 1460, 1977.

38. Mackler, B. F., Waldrop, T. C., Schur, P., et al.: IgG subclasses in human periodontal disease. 1. Distribution and incidence of IgG subclasses bearing lymphocytes and plasma cells. J Periodont Res 13: 109, 1978.

39. Seymour, G. J., Powell, R. N., and Davies, W. T.: Conversion of a stable T-cell lesion to a progressive B-cell lesion in the pathogenesis of chronic inflammatory periodontal disease: an hypothesis. $J$ Clin Periodontol 6: 267, 1979.

40. Genco., R. J., and Slots, J.: Host response in periodontal disease. $J$ Dent Res 63: 441, 1984.

41. Smith, S., Bick, P. H., Miller, G. A., et al.: Polyclonal B-cell activation: severe periodontal disease in young adults. Clin Immunol Immunopathol 16: 354, 1980.

42. Zambon, J. J., Christersson, L. A., and Slots, J.: Actinobacillus actinomycetemcomitans in human periodontal disease. $J$ Periodontol 54: 707, 1983.

43. Okuda, K., Naito, Y., Ohta, K., et al.: Bacteriological study of periodontal lesions in two sisters with juvenile periodontitis and their mother. Infect Immun 45: 118, 1984.

44. Listgarten, M.A.: Structure of the microbial flora associated with periodontal health and disease in man. A light and electron microscope study. J Periodontol 47: 1, 1976.

45. Keyes, P. H., and Rams, T. E.: A rational for management of periodontal disease: rapid identification of microbial "therapeutic targets" with phase-contrast microscopy. J Am Dent Assoc 106: 803, 1983.

46. Loesche, W. J., and Laughon, B.: Role of spirochetes in periodontal disease. Host-Parasite Interactions in Periodontal Disease. R. J. Genco and S. E. Mergenhagen (eds), pp 62-75. Washington, DC, American Society for Microbiology, 1982.

47. Boehranger, H., Taichman, N. S., and Shenker, B. J.: Suppression of fibroblast proliferation by oral spirochetes. Infect Immun 45: $155,1984$.

48. Zambon, J. J., Reynolds, H. S., and Slots, J.: Black pigmented Bacteroides spp. in the human oral cavity Infect Immun 32: 198, 1981.

49. Liljenberg, B., and Lindhe, J.: Juvenile periodontitis. Some microbiological, histopathological and clinical characteristics. J Clin Periodontol 7: 48, 1980.

50. Vandesteen, G. E., Williams, B. L., Ebersole, J. L., et al.: Clinical, microbiological, and immunological studies of a family with a high prevalence of early-onset periodontitis. J Periodontol 55: 159, 1984.

51. Loesche, W. J., Gusberti, F., Mettraux, G., et al.: Relationship between oxygen tension subgingival bacterial flora in untreated human periodontal pockets. Infect Immun 42: 659, 1983.

52. Haffajee, A. D., Goodson, J. M., and Socransky, S. S.: Patterns of bone loss in patients in advanced destructive periodontal disease. J Dent Res 58A: 345, 1979.

53. Goodson, J. M.: Acute exacerbation in chronic periodontal disease. J Can Dent Assoc 13: 380, 1984.

54. Joyston-Bechal, S., Smales, F. C., and Duckworth, R.: Effect of metronidazole on chronic periodontal disease in subjects using a topically applied chlorhexidine gel. J Clin Periodontol 11: 53, 1984.

Send reprint requests to: Dr. Walter J. Loesche, Professor of Dentistry, University of Michigan School of Dentistry, Ann Arbor, MI 48109.

\section{ERRATUM}

In his article entitled: Dentinal Sensation and Hypersensitivity, A Review of Mechanisms and Treatment Alternatives which appeared in the April 1985 issue of the Journal of Periodontology, Dr. Berman misidentified the dentifrices which were compared by Tarbet et al.

The product Protect, not Promise, was a test dentifrice in the cited Tarbet reference. 